\title{
Aplicação de modelos não-lineares para descrever a evolução de características de crescimento e carcaça em bovinos da raça Hereford
}

\author{
Application of nonlinear models to describe the evolution of growth and carcass traits in \\ Hereford cattle
}

\section{Rafael Espigolan $^{\mathrm{I}}$ Fernando Baldi $^{\mathrm{II}}$ Arione Augusti Boligon ${ }^{\mathrm{I}}$ Georgget Banchero ${ }^{\mathrm{III}}$ Gustavo Brito ${ }^{\mathrm{III}}$ Alejandro La Manna ${ }^{\text {III }}$ Fabio Montossi ${ }^{\text {III }}$ Enrique Fernandez ${ }^{\mathrm{III}}$ Lucia Galvão de Albuquerque}

\section{RESUMO}

Este trabalho foi desenvolvido com o objetivo de estudar a evolução das características de crescimento e carcaça em função da idade em bovinos da raça Hereford, utilizando modelos não-lineares. Foram utilizados dados de 240 bovinos machos (castrados) da raça Hereford, pertencentes à fazenda Experimental "La Estanzuela" do INIA, Colonia, Uruguai. As características começaram a ser mensuradas a partir da desmama de animais com aproximadamente seis meses de idade, até o abate (dois anos de idade). As medições de peso foram realizadas a cada $15 \pm 3$ dias durante todo o período e sem jejum prévio. As mensurações de área de olho de lombo, obtidas por ultrassom, e altura dos animais foram obtidas a cada 90 dias, sendo que as medidas de ultrassom foram tomadas entre a $12^{\underline{a}}$ e $13^{\underline{a}}$ costelas. Os modelos não-lineares utilizados foram os modelos de Brody, Gompertz, Logístico e Von Bertalanffy. Para as características peso, altura e área de olho de lombo, todos os modelos avaliados atingiram a convergência. Para descrever a evolução dos dados de peso e área de olho de lombo em função da idade, o modelo de Von Bertalanffy foi o mais indicado, e os modelos de Brody e Logístico mostraram o pior ajuste, respectivamente. A evolução da altura dos animais desde o desmame até os dois anos de idade pode ser modelada através de qualquer um dos modelos não-lineares considerados neste estudo.

Palavras-chave: curva de crescimento, gado de corte, peso, qualidade de carne.

\section{ABSTRACT}

The objective of this research was to study the evolution of growth and carcass traits as a function of age in Hereford cattle using nonlinear models. Records from 240
Hereford steers (castrated), belonging to the experimental station "La Estanzuela" of INIA Uruguay, were utilized. The animals were measured from weaning, approximately at 6 months of age, to slaughter (two years of age). The animals were weighed, without fasting, every $15 \pm 3$ days throughout the period. The measurements of ribeye loin area (between the $12^{\text {th }}$ and $13^{\text {th }}$ rib) were obtained by ultrasound every 90 days. The height of the animals was obtained every 90 days. The Brody, Gompertz, Logistic and Von Bertalanffy nonlinear models were applied to describe the trajectory of weight, height and ribeye loin area along the age. For height, weight and ribeye loin area, all the models reached the convergence. To describe the evolution of weight and ribeye loin area, the Von Bertalanffy nonlinear model was the most suitable. For weight and ribeye loin area, the Brody and Logistic nonlinear models have shown the worst fit, respectively. The height trajectory from weaning until two years of age can be modeled by any of the nonlinear models considered in this research.

Key words: beef cattle, growth curve, meat quality, weight.

\section{INTRODUÇÃO}

No atual sistema de produção de carne, os criadores estão cada vez mais preocupados com o crescimento animal e com algumas características de carcaça, como a área de olho de lombo, pois estas representam uma relação direta com a quantidade e qualidade da carne produzida. Assim, a técnica de análise de medidas repetidas é de fundamental importância na produção animal. Essas análises incluem

'Departamento de Zootecnia, Faculdade de Ciências Agrárias e Veterinárias (FCAV), Universidade Estadual Paulista (UNESP), Jaboticabal, SP, Brasil. E-mail: espigolan@yahoo.com.br. *Autor para correspondência.

"Departamento de Nutrição e Produção Animal, Faculdade de Medicina Veterinária e Zootecnia (FMVZ), Universidade de São Paulo (USP), Pirassununga, SP, Brasil

IIIInstituto Nacional de Investigación Agropecuária (INIA), Estación Experimental La Estanzuela, Colonia, Uruguay. 
situações em que as unidades experimentais ou os indivíduos de diferentes subpopulações ou tratamentos, como sexo e raça, são estudados ao longo de diversas condições de avaliação. Dentre essas análises, sobressaem as curvas de crescimento, que relacionam o peso e a idade dos animais, por meio de modelos não-lineares (DAVIDIAN \& GILTINAN, 1996).

A utilização de modelos não-lineares, desenvolvidos para relacionar medidas de crescimento em função da idade dos animais, tem se mostrado adequada para descrever a curva de crescimento, uma vez que essas funções sintetizam um grande número de medidas em apenas alguns parâmetros com significado biológico, facilitando, dessa forma, a interpretação e o entendimento do fenômeno (BROWN et al., 1976). O ajuste de curvas de crescimento é feito usando uma medida de tamanho, como o peso corporal, comprimento ou altura e idade em uma conveniente unidade de tempo. Desse modo, este trabalho teve por objetivo estudar a evolução das características de crescimento e carcaça em função da idade em bovinos da raça Hereford, utilizando modelos não-lineares (Brody, Gompertz, Logístico e Von Bertalanffy).

\section{MATERIAL E MÉTODOS}

O presente trabalho foi desenvolvido com dados de 240 bovinos machos (castrados) da raça Hereford, provenientes de um único rebanho, filhos de vacas multíparas e pertencentes à fazenda Experimental "La Estanzuela" do Instituto Nacional de Investigación Agropecuária (INIA), Colonia, Uruguai. As características começaram a ser mensuradas a partir da desmama, com animais de aproximadamente seis meses de idade, sendo obtidas até o abate (dois anos de idade).

As medições de peso foram realizadas a cada $15 \pm 3$ dias durante todo o período e sem jejum prévio. As mensurações de área de olho de lombo, obtidas por ultrassom, e altura dos animais foram obtidas a cada 90 dias, desde o desmame até o abate. As medidas de ultrassom foram tomadas entre as $12^{a}$ e $13^{\text {a }}$ costelas, feitas por meio de um ultrassom da marca Aloka 500 e um transdutor de $17 \mathrm{~cm}$, com frequência de $3,5 \mathrm{MHz}$. A altura dos animais foi obtida entre a cernelha e o solo.

Após a desmama, os animais foram manejados sobre pastagem de aveia (Avena sativa) ou em confinamento, até os 10 meses de idade. Posteriormente, os animais permaneceram juntos, com manejo similar sobre pastagens de alfafa (Medicago sativa) até atingirem a média de $350 \mathrm{~kg}$ de peso vivo. Em seguida, metade dos novilhos continuaram sobre pastagem de alfafa e a outra parte foi terminada em condições de confinamento. $\mathrm{O}$ abate dos animais foi realizado quando a média de peso de cada grupo de terminação atingiu $500 \mathrm{~kg}$, com idades que variaram entre 18 a 24 meses.

Após a consistência dos dados, foram obtidas as estatísticas descritivas (número de observações, média, desvio-padrão, valores mínimo e máximo e coeficiente de variação) para cada uma das características mensuradas (Tabela 1). Na consistência dos dados, foram eliminadas as observações (outliers) com desvios importantes em relação à média $(3,0$ desvios-padrão acima ou abaixo da média) para cada idade considerada. Os modelos não-lineares utilizados (Brody, Gompertz, Logístico e Von Bertalanffy) são representados pelas seguintes expressões:

Brody: $\mathrm{Wt}=\mathrm{A}\left(1-\mathrm{b} \mathrm{e}^{-\mathrm{kt}}\right)$

Gompertz: $\mathrm{Wt}=\mathrm{A} \exp \left(-\mathrm{b} \mathrm{e}^{-\mathrm{kt}}\right)$

Logístico: $\mathrm{Wt}=\mathrm{A} /\left(1+\mathrm{be}^{-\mathrm{kt}}\right)$

Von Bertalanffy: $\mathrm{Wt}=\mathrm{A}\left(1-\mathrm{b} \mathrm{e}^{-\mathrm{kt}}\right)^{3}$

Nessas funções, no caso da característica evolução de peso, Wt (kg) é o peso na idade t (dias); A $(\mathrm{kg})$ é o peso assintótico quando a idade $\mathrm{t}$ tende ao infinito; b é o peso inicial e $\mathrm{k}$ é a taxa de crescimento (maturidade). A interpretação dos parâmetros das funções são as seguintes (SARMENTO et al., 2006): parâmetro A - definido como o valor assintótico do tecido ou característica, representa o valor máximo que característica pode atinigir na maturidade; parâmetro b é uma constante de integração, relacionado aos valores iniciais da característica, indicando a proporção do crescimento assintótico a ser obtida depois do nascimento, estabelecidos pelos valores iniciais da característica e o tempo; parâmetro $\mathrm{k}$ - corresponde ao índice de maturidade e determina a eficiência do crescimento do animal, indicando a velocidade de crescimento do tecido até a idade adulta.

Para ajustar os diferentes modelos nãolineares, foi utilizado o procedimento NLIN, disponível no software SAS (2008). Estimativas dos parâmetros de

Tabela 1 - Estatísticas descritivas para as características peso, altura e área de olho de lombo e respectivos números de observações.

\begin{tabular}{|c|c|c|c|c|c|c|}
\hline Características & Número de observações & Média & Desvio-padrão & Máximo & Mínimo & Coeficiente de variação \\
\hline Peso (kg) & 6.810 & 319,9 & 104,9 & 592,0 & 124,0 & 32,8 \\
\hline Altura $(\mathrm{cm})$ & 1.797 & 117,7 & 9,9 & 139,5 & 93,0 & 8,5 \\
\hline Área de olho de lombo $\left(\mathrm{cm}^{2}\right)$ & 1.622 & 46,3 & 11,4 & 81,9 & 21,5 & 24,6 \\
\hline
\end{tabular}

Ciência Rural, v.43, n.3, mar, 2013. 
crescimento foram obtidas usando o método de GaussNewton, modificado no procedimento NLIN do SAS (TOPAL et al., 2004). Os critérios adotados para selecionar o modelo que melhor descreveu o comportamento das características foram (FREITAS et al., 2005): a) convergência ou não das análises, b) quadrado médio do erro e coeficiente de determinação do modelo; e c) interpretação biológica dos parâmetros. A interpretação biológica dos parâmetros consiste na comparação entre o valor predito pelos modelos e os dados observados, bem como com valores observados na literatura para determinada característica em certa idade e raça. Portanto, quando as diferenças entre os parâmetros e entre os quadrados médios do erro são pequenas para dois ou mais modelos não-lineares, o critério de interpretação biológica dos parâmetros é uma ferramenta viável para escolher o modelo mais adequado.

Nas análises realizadas no presente estudo, foram desconsiderados os manejos nutricionais durante a recria e a terminação dos animais, em virtude da maior subdivisão dos tratamentos e, portanto, diminuição do número de animais por tratamento (quatro manejos na recria $\mathrm{x}$ dois tipos de terminação = oito tratamentos). Análises preliminares foram realizadas, mas a convergência ficou muitas vezes comprometida quando foram considerados os oito manejos alimentares.

\section{RESULTADOS E DISCUSSÃO}

Com todos os modelos avaliados, foi atingida a convergência das análises (Tabela 2). De acordo com o quadrado médio do erro, o modelo não- linear de Brody mostrou o pior ajuste para a evolução de peso. $\mathrm{O}$ coeficiente de determinação foi próximo para todos os modelos avaliados. O modelo não-linear de Von Bertalanffy apresentou valores para o quadrado médio do erro menor, indicando um melhor ajuste. Os modelos de Gompertz e Von Bertalanffy estimaram valores de peso assintótico superiores (parâmetro $A$ ) em relação ao modelo Logístico. Porém, de certa forma, todos os modelos não-lineares estudados, exceto o modelo de Brody, foram adequados para descrever a evolução do peso da desmama até os dois anos de idade em novilhos da raça Hereford. Como o parâmetro A representa o peso adulto ou a estimativa de peso à maturidade ou no infinito, admite-se que um novilho da raça Hereford sem restrição alimentar e a uma idade mais avançada, na maturidade, atinja pesos variando de 600 a 900kg (MAZZINI et al., 2005).

Em relação ao parâmetro b, SARMENTO et al. (2006) afirmam que esse parâmetro representa uma constante de integração, relacionada aos pesos iniciais do animal e sem interpretação biológica bem definida, portanto, este parâmetro não foi considerado na seleção dos modelos não-lineares. A estimativa do parâmetro k está relacionada com a taxa de maturação dos animais. Estimativas maiores do que as obtidas no presente estudo para o parâmetro $\mathrm{k}$ foram relatadas por OLIVEIRA et al. (2000), que trabalharam com animais da raça Guzerá, com pesos do nascimento até os 36 meses de idade.

Na figura 1, é apresentada a evolução dos valores médios observados e preditos para peso em função da idade, obtidos com a utilização de modelos

Tabela 2 - Estimativas dos parâmetros dos modelos não-lineares de Brody, Gompertz, Logístico e Von Bertalanffy, coeficiente de determinação $\left(\mathrm{R}^{2}\right)$ e quadrado médio do erro (QME) para peso, altura e área de olho de lombo.

\begin{tabular}{llccccc}
\hline Características & Modelo & $\mathrm{A}^{1}$ & $\mathrm{~b}$ & $\mathrm{k}$ & $\mathrm{R}^{2}$ & $\mathrm{QME}$ \\
\hline \multirow{3}{*}{ Peso $(\mathrm{kg})$} & Brody & 623,70 & 0,7886 & 0,00212 & 0,9578 & 1485,80 \\
& Gompertz & 779,50 & 1,6002 & 0,00255 & 0,9675 & 1211,00 \\
& Logístico & 624,00 & 2,8655 & 0,00478 & 0,9673 & 1218,20 \\
& Von Bertalanffy & 908,20 & 0,4441 & 0,00180 & 0,9676 & 1209,50 \\
& & & & & & \\
& Brody & 131,50 & 0,2064 & 0,00715 & 0,9749 & 12,15 \\
Altura $(\mathrm{cm})$ & Gompertz & 131,20 & 0,2278 & 0,00782 & 0,9741 & 12,17 \\
& Logístico & 130,90 & 0,2471 & 0,00845 & 0,9734 & 12,23 \\
& Von Bertalanffy & 131,30 & 0,0730 & 0,00773 & 0,9740 & 12,20 \\
& & & & & & \\
Área de olho de lombo $\left(\mathrm{cm}^{2}\right)$ & Brody & 155,30 & 0,8213 & 0,000647 & 0,8997 & 32,03 \\
& Gompertz & 94,52 & 1,2035 & 0,00219 & 0,9012 & 31,90 \\
& Logístico & 80,45 & 1,8123 & 0,00364 & 0,9023 & 32,10 \\
& Von Bertalanffy & 103,40 & 0,3530 & 0,00167 & 0,9007 & 31,10 \\
\hline
\end{tabular}

${ }^{1} \mathrm{~A}$ : valor da característica à maturidade; b: constante de integração; k: índice de maturidade 


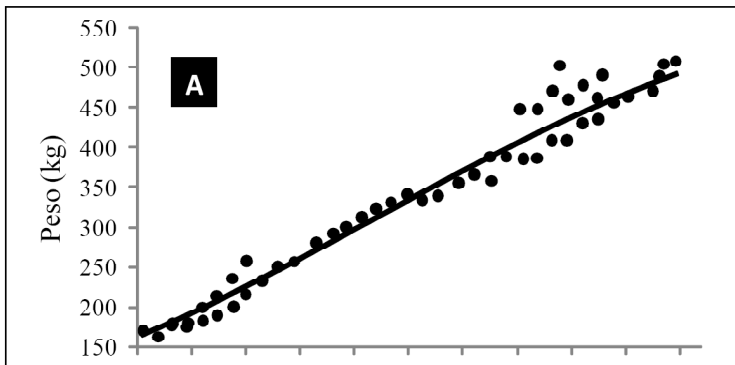

$\begin{array}{lllllllllll}180 & 230 & 280 & 330 & 380 & 430 & 480 & 530 & 580 & 630 & 680\end{array}$ Idade (dias)

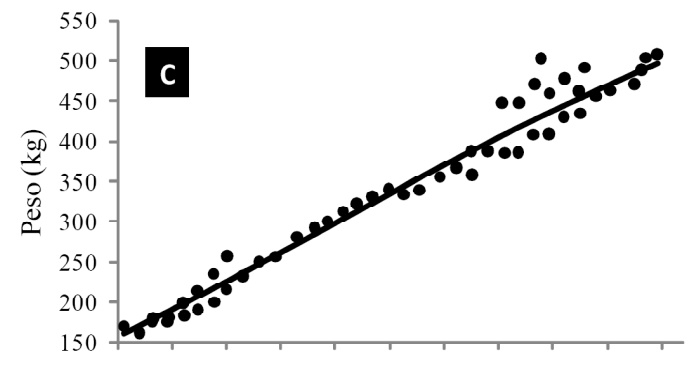

$\begin{array}{lllllllllll}180 & 230 & 280 & 330 & 380 & 430 & 480 & 530 & 580 & 630 & 680\end{array}$ Idade (dias)
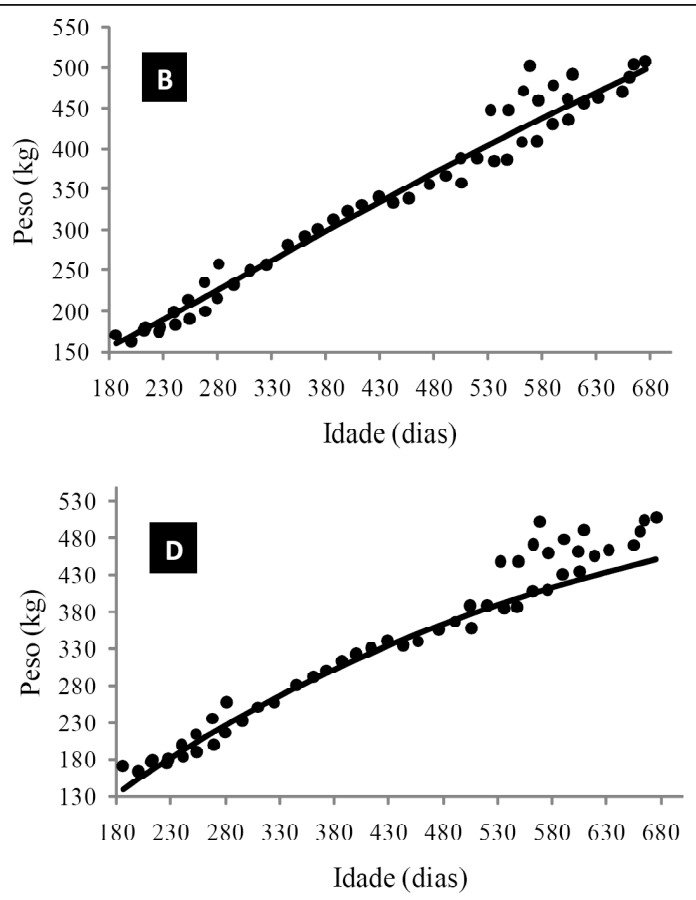

Figura 1 - Evolução dos valores médios de peso observados $(\bullet)$ e preditos através dos modelos não-lineares Logístico (A), Von Bertalanffy (B), Gompertz (C) e Brody (D).

não-lineares. Nas figuras 1-A, 1-B e 1-C, pode ser observado que os modelos não-lineares Logístico, Gompertz e Von Bertalanffy mostraram uma curva de valores preditos semelhante e bem próxima aos dados observados. Entretanto, a função de Brody (Figura 1D), que, de acordo com o quadrado médio do erro, proporcionou o pior ajuste, mostrou maior discrepância entre os valores médios observados e preditos, sobretudo para os pesos a partir de 500 dias de idade. MAZZINI et al. (2005) utilizaram os modelos de Brody, Gompertz, Logístico e Von Bertalanffy para descrever a evolução de pesos de 152 novilhos da raça Hereford do nascimento até 720 dias de idade, sendo que os modelos de Gompertz e Von Bertalanffy apresentaram melhor ajuste. BERGAMASCO et al. (2001) utilizaram os modelos Logístico, Brody e Gompertz para descrever o crescimento (peso em função do tempo) de 82 fêmeas da raça Holandesa do nascimento até os dois anos de idade, sendo que os modelos Logístico e Gompertz apresentaram a melhor qualidade de ajuste.

Para descrever a evolução da altura em função da idade, todos os modelos avaliados atingiram a convergência das análises (Tabela 2). O quadrado médio do erro e o coeficiente de determinação para as funções de Brody, Gompertz, Logístico e Von Bertalanffy foram próximos, indicando que todos os modelos tiveram um ajuste semelhante. Utilizando informações de 26 cavalos Pantaneiros, SANTOS et al. (1999) ajustaram dados de altura da cernelha em função da idade (do nascimento aos 36 meses de idade), aplicando os modelos não-lineares de Brody, Richards, Gompertz, Logístico, Weibull e Morgan-Mercer-Flodin. De acordo com os autores, ao considerar os critérios de erro médio quadrático e medida de curvatura média, o modelo de Weibull foi o mais adequado para modelar o crescimento em altura para cavalos Pantaneiros.

Para todos os modelos não-lineares avaliados, foram estimados valores muito próximos para o parâmetro A (Tabela 2). Essa proximidade nas estimativas de valores para o parâmetro relacionado com altura do animal à maturidade pode ser explicada pelo fato de a altura ser uma característica de maturação precoce, uma vez que está associada ao desenvolvimento ósseo. Segundo DI MARCO (1998), a altura constitui uma característica que atinge um patamar de estabilidade precocemente, pois o crescimento em altura dos animais depende, fundamentalmente, entre outros fatores, do crescimento do tecido ósseo.

Os valores preditos para a altura, utilizando os modelos não-lineares avaliados neste trabalho, 
apresentaram um comportamento semelhante em função da idade e estiveram bem próximos dos valores médios observados (Figura 2). Como pode ser observado na figura 2-A, figura 2-B, figura 2-C e figura 2-D, a altura mostra ser uma característica que atinge um patamar precocemente e, assim, permanece praticamente constante a partir dos 18 meses de idade, demonstrando seu comportamento assintótico, fato que facilita o ajuste por meio dos modelos não-lineares. Como os dados utilizados no presente estudo foram obtidos desde o desmame até os dois anos de idade, nesse período, provavelmente, os animais já haviam alcançado ou estavam próximos do máximo crescimento ósseo, em torno dos 580 dias de idade, dado o comportamento assintótico das curvas apresentadas na figura 2. Portanto, os resultados obtidos indicam que todos os modelos testados foram adequados para descrever a evolução da altura em animais da raça Hereford até dois anos de idade.

Para a área de olho de lombo, todos os modelos não-lineares avaliados atingiram a convergência das análises (Tabela 2). O coeficiente de determinação obtido para todos os modelos avaliados foi semelhante. De acordo com o quadrado médio do erro, o modelo Logístico foi aquele que proporcionou o pior ajuste. Entretanto, o modelo de Von Bertalanffy proporcionou o menor quadrado médio do erro, indicando um melhor ajuste.
No presente estudo, a estimativa do parâmetro A obtida pelo modelo Von Bertalanffy foi próxima da descrita por TÖRÖK et al. (2009), que relataram área de olho de lombo de $102,9 \mathrm{~cm}^{2}$, para touros da raça Angus aos 18 meses de idade em condições de confinamento. Trabalhando com dados de 2.590 bovinos da raça Nelore, YOKOO et al. (2009) relataram valores médios para área de olho de lombo obtida ao sobreano de $48,4 \mathrm{~cm}^{2}$.

O modelo Logístico proporcionou valor do parâmetro $\mathrm{k}$ (índice de maturidade) maior em comparação com os outros modelos não-lineares (Tabela 2), denotando um intenso crescimento muscular durante o período considerado. No caso do modelo não-linear de Brody, a estimativa do parâmetro $A$ foi superestimada em relação aos valores médios observados, comprometendo as predições obtidas a partir desse modelo.

Na figura 3, é apresentada a evolução dos valores médios observados e dos valores preditos para área de olho de lombo por meio dos modelos não-lineares Logístico, Von Bertalanffy, Gompertz e Brody. No entanto, os modelos Logístico, Gompertz e Von Bertalanffy apresentaram um comportamento semelhante e os valores preditos estiveram próximos dos valores médios observados. Cabe destacar que a utilização de modelos não-lineares para a descrição da evolução das características da carcaça são raras na literatura.
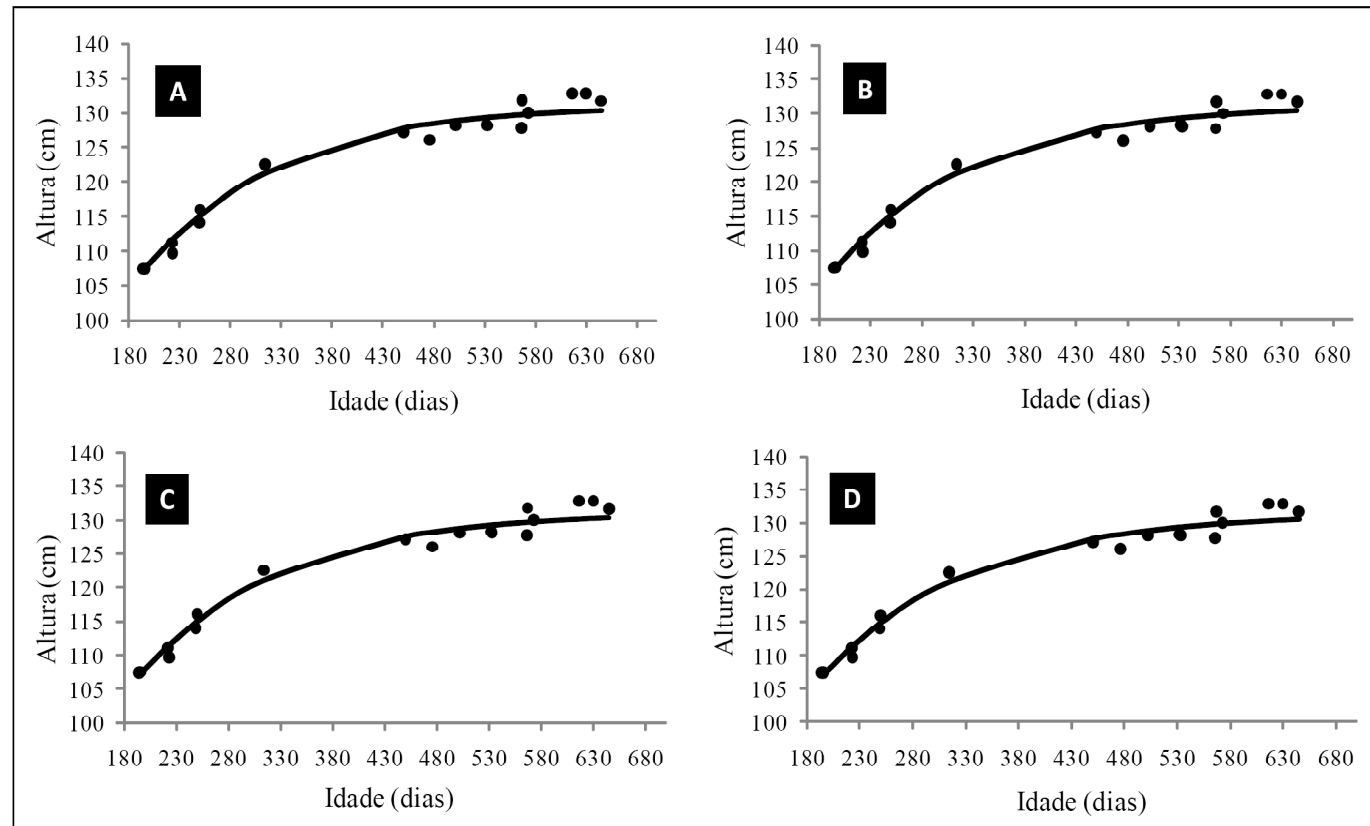

Figura 2 - Evolução dos valores médios de altura observados $(\bullet)$ e preditos através dos modelos não-lineares Logístico (A), Von Bertalanffy (B), Gompertz (C) e Brody (D).

Ciência Rural, v.43, n.3, mar, 2013. 


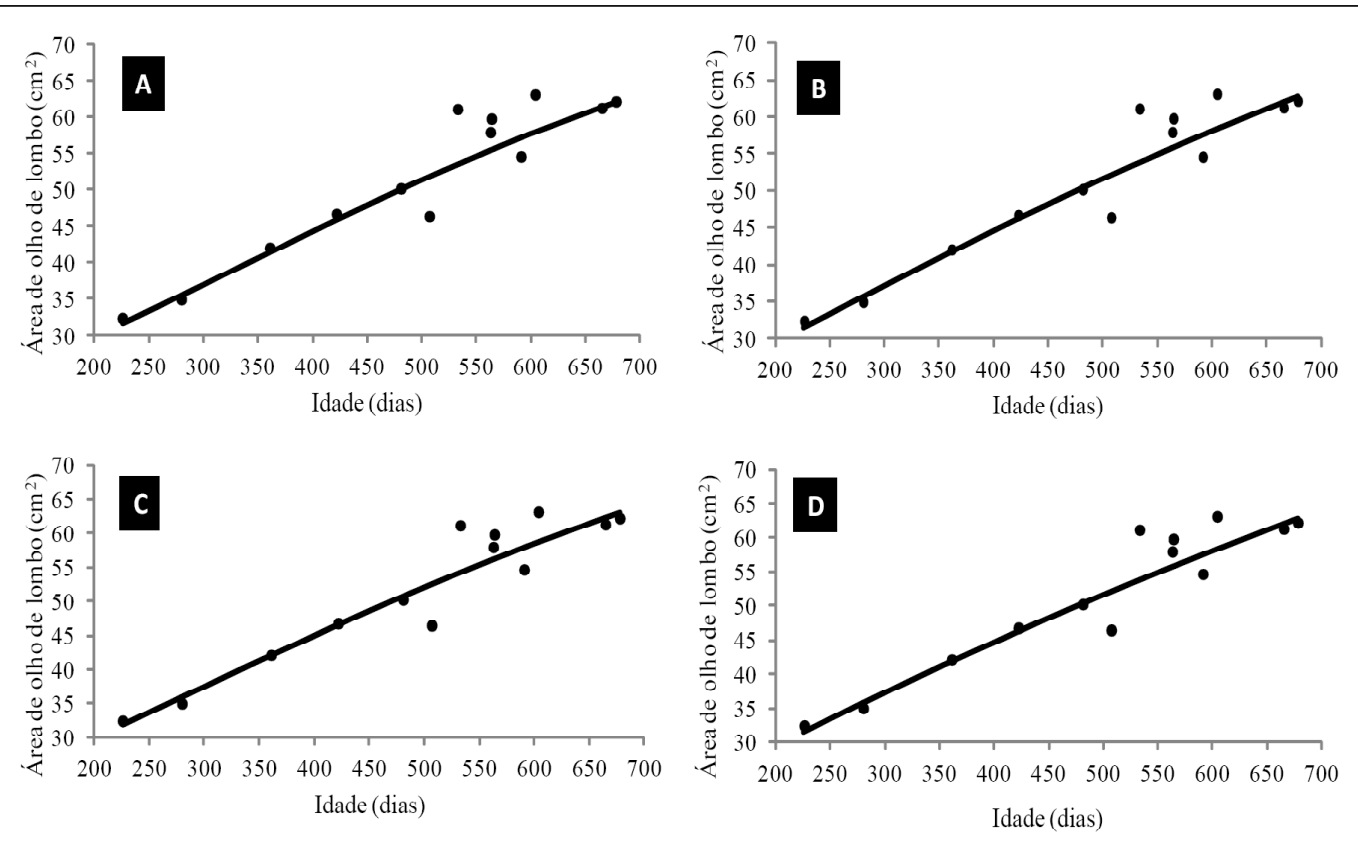

Figura 3 - Evolução dos valores médios para área de olho de lombo observados $(\bullet)$ e preditos pelos modelos nãolineares Logístico (A), Von Bertalanffy (B), Gompertz (C), Brody (D).

\section{CONCLUSÃO}

Para descrever a evolução de peso e área de olho de lombo em novilhos da raça Hereford da desmama até os dois anos de idade, o modelo de Von Bertalanffy é o mais indicado. A evolução da altura pode ser modelada através de qualquer um dos modelos não-lineares considerados no presente estudo.

\section{AGRADECIMENTOS}

Ao Instituto Nacional de Investigación Agropecuária (INIA), Colonia, Uruguai, pelo apoio financeiro, à Sociedad Criadores de Hereford del Uruguay, e à Fundação de Amparo a Pesquisa do Estado de São Paulo (FAPESP) pela concessão da bolsa de iniciação científica, Projeto $\mathrm{N}^{\circ}$ 2009/08058-2.

\section{REFERÊNCIAS}

BERGAMASCO, A.F. et al. Ajuste de modelos não-lineares a dados de crescimento de fêmeas da raça Holandesa. Ciência e Agrotecnologia, v.25, p.235-241, 2001. Disponível em: <http:/ /www.editora.ufla.br/site/revista_detalhes.php?r=25\&n=54>. Acesso em: 23 jan. 2012.

BROWN, J.E. et al. A comparison of nonlinear models for describing weight-age relationships in cattle. Journal of Animal Science, v.42, p.810-818, 1976. Disponível em: <http://www.journalofanimalscience.org/content/42/4/810>. Acesso em: 14 fev. 2011.
DAVIDIAN, M.; GILTINAN, D.M. Nonlinear models for repeated measurement data. 2.ed. London: Chapman Hall, 1996. 359p.

DI MARCO, O.N. Crecimiento de vacunos para carne. Mar Del Plata: edição do autor, 1998. 246p.

FREITAS, A.R. et al. Alternativas de análises em dados de medidas repetidas de bovinos de corte. Revista Brasileira de Zootecnia, v.34, p.2233-2244, 2005. Disponível em: <http:/ $/$ www.scielo.br/scielo.php?script=sci_arttext\&pid=S1516359 $82005000700010 \& \operatorname{lng}=\mathrm{pt} \& \mathrm{nrm}=\mathrm{iso} \& \mathrm{t} \operatorname{lng}=\mathrm{pt}>$. Acesso em: 01 jul. 2011. doi: 10.1590/S1516-35982005000700010.

MAZZINI, A.R.A. et al. Curva de crescimento de novilhos Hereford: heterocedasticidade e resíduos autorregressivos. Ciência Rural, v.35, p.422-427, 2005. Disponível em: <http:/ /www.scielo.br/scielo.php?pid=S0103-8478200500 $0200028 \&$ script=sci_arttext $>$. Acesso em: 19 set. 2011. doi: 10.1590/S0103-84782005000200028.

MEZZADRA, C.A. et al. Comparación del desempeño productivo de novillos puros y cruza británicos bajo sistemas de engorde semi-intensivos e intensivos. Revista Argentina de Producción Animal, v.23, p.45-52, 2003. Disponível em: <http://www.aapa.org.ar/archivos/revistas/2003/vol23n1/ 005GMMezzadra.pdf>. Acesso em: 28 set. 2011.

OLIVEIRA, H.N. et al. Comparação de modelos não-lineares para descrever o crescimento de fêmeas da raça Guzerá. Pesquisa Agropecuária Brasileira, v.35, n.9, p.1843-1851, 2000. Disponível em: <http://www.scielo.br/pdf/pab/v35n9/ v35n9a17.pdf>. Acesso em: 29 set. 2011. doi: 10.1590/S0100204X2000000900017. 
SANTOS S.A. et al. Using nonlinear models to describe height growth curves in Pantaneiro horses. Pesquisa Agropecuária Brasileira, v.34, p.1133-1138, 1999. Disponível em: <http:/ /www.scielo.br/scielo.php?pid=S0100-204X1999000700003 $\&$ script=sci_arttext $>$. Acesso em: 27 set. 2011. doi: 10.1590/ S0100-204X1999000700003.

SARMENTO, J. L. R. et al. Estudo da curva de crescimento de ovinos Santa Inês. Revista Brasileira de Zootecnia, v.35, p.435-442, 2006. Disponível em: <http://www.scielo.br/pdf/rbz/ v35n2/a14v35n2.pdf>. Acesso em: 13 jul. 2011. doi: 10.1590/ S1516-35982006000200014.

SAS (STATISTICAL ANALYSIS SYSTEM). User's guide. Version 9.2. 2.ed. Cary, 2008. (CD-ROM).

TOPAL, M.; OZDEMIR, M.; AKSALKAL, V.; YILDIZ, N.; DOGRU, U. Determination of the best nonlinear function in order to estimate growth in Morkaramam and Awassi lambs.
Small Ruminant Research, v.55, p.229-232, 2004. Disponível em: <http://www.sciencedirect.com/science/article/ pii/S0921448804000513 >. Acesso em: 14 ago. 2011. doi: 10.1016/j.smallrumres.2004.01.007.

TÖRÖK M. J. et al. Correlation of ultrasonic measured ribeye area and fat thickness to the certain traits measured on slaughtered bulls. Archiv Tierzucht / Archives Animal Breeding, v.1, p.23-27, 2009. Disponível em: <http://archan im-breed.fbn-dummerstorf.de/content/2009/ cont2009.html>. Acesso em: 14 ago. 2011.

YOKOO, M. J. I. et al. Correlações genéticas entre escores visuais e características de carcaça medidas por ultrassom em bovinos de corte. Pesquisa Agropecuária Brasileira, v.44, p.197-202, fev. 2009. Disponível em: <http://www.scielo.br/ pdf/pab/v44n2/v44n02a12.pdf $>$. Acesso em: 10 jul. 2011. doi: 10.1590/S0100-204X2009000200012. 